\title{
Desenvolvimento de manual para orientaçóes básicas do dia a dia para pessoas com esclerose múltipla ${ }^{1}$
}

\author{
Paula Pozzi Pimentel, Rosé Colom Toldrá \\ Departamento de Fisioterapia, Fonoaudiologia e Terapia Ocupacional, Universidade de São Paulo - USP, \\ São Paulo, SP, Brasil.
}

\begin{abstract}
Resumo: Objetivo: O estudo visa apresentar as etapas desenvolvidas para o aprimoramento de manual de orientação e sua aplicação com pessoas com esclerose múltipla (EM), ocorrida em um grupo terapêutico conduzido pela Terapia Ocupacional. Conforme as políticas de saúde, a produção de materiais educativos tem caráter multidisciplinar e é útil como ferramenta para a promoção de saúde devido à sua capacidade de aumentar o conhecimento e de solucionar problemas. O caráter crônico e progressivo da EM traz alterações em diferentes funções do corpo e demanda mudanças e estratégias para minimizar o seu impacto na vida das pessoas. Método: Estudo documental baseado nos registros escritos e áudios produzidos nos encontros grupais no período de 2013 a 2014. A análise dos documentos foi feita com base no método do Discurso do Sujeito Coletivo (DSC). Resultados: Participaram do estudo 10 pessoas com EM, com idade entre 23 a 55 anos, a maioria com mais de cinco anos de doença. Foram gerados quatro DSCs. De acordo com os discursos, o manual serviu não só como uma ferramenta prática e de fácil consulta, o que favoreceu a desmistificação da doença, mas também como guia para as atividades da rotina, apoio para identificação dos problemas e facilitação na convivência com a rede social. Conclusão: $\mathrm{O}$ uso do manual mostrou-se útil por facilitar a prática do aprendizado adquirido no grupo e estimular a corresponsabilização com a própria saúde.
\end{abstract}

Palavras-chave: Esclerose Múltipla, Atenção à Saúde, Educação em Saúde, Manual, Orientação, Terapia Ocupacional.

\section{Manual development for basic guidelines of the day-to-day for people with multiple sclerosis}

\begin{abstract}
Objective: The study aims to present the steps developed for the guidance manual enhancement and its application on people with multiple sclerosis occurred in a therapeutic group conducted by the Occupational Therapy. According to health policies, the production of educational materials is multidisciplinary and is useful as a tool to promote health due to this ability to increase the knowledge and problem solutions. The chronic and progressive disease brings alterations in different body functions and demands changes and strategies to minimize their impact on people's lives. Method: Documentary study based on written records and audio produced in the group meetings in the period from 2013 to 2014. The analysis of the documents was based on the Collective Subject Discourse (CSD) method. Results: The study included 10 people with multiple sclerosis, aged 23-55 years, the majority with over 5 years of disease. It was generated four CSD. According to the speeches the manual served as a practical tool and easy consultation, which favored demystify the disease, served as a guide for the routine activities, support identifying problems and facilitating the interaction with the social network. Conclusion: The use of the manual was useful because it facilitates the practice of acquired skills in the group and encourage co-responsibility for their own health.
\end{abstract}

Keywords: Multiple Sclerosis, Health Care, Education in Health, Manual, Guidance, Occupational Therapy.

Autor para correspondência: Rosé Colom Toldrá, Departamento de Fisioterapia, Fonoaudiologia e Terapia Ocupacional, Universidade de São Paulo, Rua Cipotânea, 51, Butantã, CEP 05360-160, São Paulo, SP, Brasil, e-mail: rosetoldra@usp.br

Recebido em Jan. 4, 2016; $1^{\text {a }}$ Revisão em Mar. 31, 2016; 2a Revisão em Maio. 21, 2016; Aceito em Jul. 7, 2016. 


\section{Introdução}

O papel da comunicação e da troca de informação durante o processo terapêutico, especialmente para as doenças crônicas, é fundamental, uma vez que o seu processo de cuidado náo depende exclusivamente de medicamentos, mas também das informaçôes que as pessoas têm sobre suas doenças e como irão desenvolver as várias habilidades necessárias para o seu enfrentamento e cuidado. Nesse sentido, a comunicação em saúde é reconhecida como uma ferramenta de promoção da saúde pela capacidade de aumentar o conhecimento e a consciência dos problemas e das soluçôes, de influenciar e promover mudanças de crenças, comportamentos sociais, assim como desenvolver atitudes e habilidades (MOREIRA; NÓBREGA; SILVA, 2003).

Para as pessoas com esclerose múltipla (EM), a informaçáo sobre a doença tem papel essencial pela heterogeneidade dos sintomas devido ao curso súbito e imprevisível da enfermidade e pela sua evolução crônica e progressiva. Isso também reafirma que a informação sobre os seus cuidados adquire um caráter ainda mais fundamental.

A EM é uma doença do sistema nervoso central, decorrente de processo inflamatório na substância branca, resultando na formação de tecidos cicatriciais, denominados de placas escleróticas, as quais impedem a propagação adequada do impulso nervoso, o que ocasiona perdas permanentes ou transitórias em qualquer função do sistema nervoso central (CALLEGARO; LINO; MARCHIORI, 2009).

A doença se caracteriza por um curso heterogêneo e imprevisível, com sintomas que variam conforme a área afetada, dentre os principais: fraqueza muscular, fadiga, alteraçóes cognitivas, dor, espasticidade, depressão e alterações visuais, esfincterianas e de sensibilidade (CALLEGARO; LINO; MARCHIORI, 2009).

É considerada a doença neurológica mais frequente em adultos jovens, acometendo pessoas entre 15 e 45 anos de idade, com maior incidência aos 30 anos (MENDES et al., 2000; FRAGOSO et al., 2010). Ocorre em uma das fases mais complexas e dinâmicas da vida, que corresponde ao desenvolvimento profissional e produtivo, podendo acarretar significativas incapacidades na realização dessas atividades (NOGUEIRA et al., 2009; TOLDRÁ, 2005) e levar à interrupção daquelas relacionadas à profissão (GARCIA-BURGUILLO; AGUILAMATURANA, 2009).

Tal condiçáo representa um desafio para o indivíduo e seus familiares, pois requer de todos os envolvidos a capacidade de constantemente aprender a conviver com as perdas gradativas das funções e as consequentes repercussões e mudanças de estilo de vida. Também aos profissionais de saúde gera grandes desafios para o desenvolvimento de práticas de atenção articuladas às necessidades específicas dessas pessoas (HASSELKUS, 2002), pois demanda o desenvolvimento contínuo de estratégias que minimizem as perdas e seu impacto no cotidiano das pessoas com EM.

Para isso, na atenção à saúde, a construção de uma relação terapêutica entre profissional e usuário deve favorecer trocas, compartilhamentos e a disponibilidade por parte do profissional para "liberar saberes e atos cuidadores", e por parte do usuário, "o desejo em restabelecer sua autonomia" (MALTA; MERHY, 2010). Esse processo envolve o fornecimento de informaçóes e o suporte ao usuário e à família para motivar escolhas responsáveis, possibilidades de qualidade de vida e independência nos atos de saúde.

Nesse sentido, a produção de cadernos, cartilhas e manuais com informaçôes sobre medidas de prevenção e de cuidado tem sido apontada como ferramenta importante pelo Ministério da Saúde nos diferentes níveis de atenção (BRASIL, 2012, 2014). Assim, o desenvolvimento de materiais educativos se mostra como uma estratégia que contribui fortemente na promoção de saúde, na autonomia e na corresponsabilidade dos sujeitos e das coletividades, no cuidado e na busca de alternativas para melhoria da saúde (BRASIL, 2012, 2014). É notável um crescente uso de materiais de orientações como recursos na educação em saúde, no contexto de atenção às doenças crônicas, por sua potência no processo de ensino-aprendizagem (TORRES et al., 2009), o que indica uma forte aplicabilidade para as pessoas com EM.

A construção e a utilização de materiais educativos envolvem todas as profissóes de saúde por ser considerada uma ferramenta útil, prática, necessária e que contribui para a complementação dos procedimentos terapêuticos. Tais materiais promovem a capacitaçáo das pessoas, colaboram na uniformização das orientações e estimulam a ação profissional. Como estratégias de apoio terapêutico, devem estar fundamentados em bases científicas e conter propostas de atividades e orientações que busquem recuperar, desenvolver ou reforçar as capacidades física, mental e social (OLIVEIRA; FERNANDES; SAWADA, 2008; ZOMBINI; PELICIONI, 2011). A utilização de materiais educativos auxilia o trabalho da equipe com os usuários e seus familiares, uma vez que reforça as informações e orientações orais, serve como guia em situação de dúvidas e, ainda, colabora para a tomada de decisóes (MOREIRA; 
NÓBREGA; SILVA, 2003; PANOBIANCO et al., 2009; TORRES et al., 2009). Para conhecer a eficácia e a eficiência dos materiais educativos, é recomendado fazer um ensaio antes de seu uso (OLIVEIRA; FERNANDES; SAWADA, 2008).

O presente estudo visa apresentar as etapas desenvolvidas para o aprimoramento de manual de orientaçôes e sua aplicação com pessoas com EM, ocorrida em um grupo terapêutico conduzido pela Terapia Ocupacional.

\section{Método}

Trata-se de estudo qualitativo, do tipo descritivo (GIL, 2006), aprovado pelo Comitê de Ética em Pesquisa da Faculdade de Medicina da Universidade de São Paulo (FMUSP), sob protocolo no 243/11. Todos os participantes da pesquisa assinaram o Termo de Consentimento Livre e Esclarecido após ciência e concordância.

Para o desenvolvimento do presente estudo, partiu-se da versão preliminar de manual de orientaçôes elaborado pela Terapia Ocupacional. A versão preliminar foi realizada em estudo anterior a partir das informaçôes obtidas com 115 pessoas com EM acompanhadas pelo Instituto Central do Hospital das Clínicas (IC-HC) da FMUSP, a qual foi considerada válida pela equipe médica desse serviço.

As pessoas possuíam o tipo da doença Remitente Recorrente e foram avaliadas por meio da Escala Expandida do Estado de Incapacidade de Kurtzke (EDSS) de 0 a 5,0, nível que corresponde a nenhuma incapacidade até incapacidades moderadas, que afetam as atividades diárias, porém com autonomia para a sua realização (CALLEGARO; LINO; MARCHIORI, 2009).

O aprimoramento do manual ocorreu como uma das atividades do "Grupo de Promoção da Saúde de Pessoas com Esclerose Múltipla”, cujo desenvolvimento se deu pela Terapia Ocupacional no Centro de Docência e Pesquisa do Departamento de Fisioterapia, Fonoaudiologia e Terapia Ocupacional da FMUSP.

Para a composição do grupo, foi realizado convite por telefone a partir de consulta ao banco de dados composto pelos 115 usuários do IC-HC da FMUSP, que contribuíram para a elaboração da versão preliminar de manual de orientaçóes.

O grupo se reuniu uma vez por semana, com um total de 22 encontros, com tempo de duração de 1 h30 cada encontro, durante seis meses, no período de 2012 a 2013. No grupo, foram realizadas a leitura comentada da versão preliminar do manual, a análise de sua aplicabilidade, o aprimoramento do manual e a sua avaliação. Durante essas etapas, procurou-se conjugar informaçôes técnicas da área com a perspectiva das pessoas com EM. A Figura 1 representa as etapas desenvolvidas.

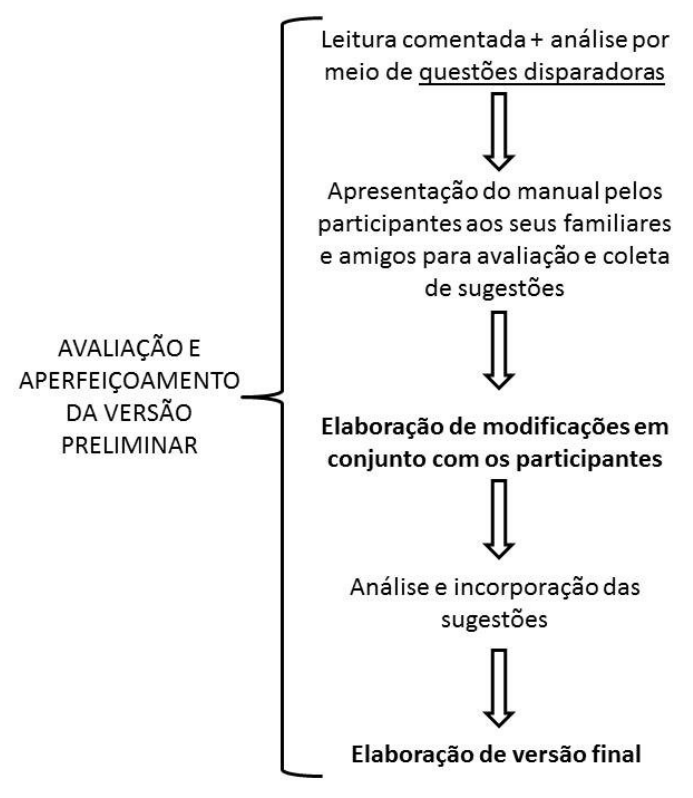

Figura 1. Etapas do processo de aprimoramento do manual de orientação, São Paulo, 2016.

Para a realização do presente trabalho, foi adotado o estudo documental referente aos registros escritos e os originados a partir da transcrição dos áudios, correspondentes aos encontros grupais desenvolvidos. Os registros escritos produzidos no grupo foram utilizados como documentos da pesquisa, em conformidade com Lüdke e André (1988). Esses autores consideram como recurso para estudo documental qualquer material escrito usado como fonte de informação referente ao comportamento humano.

Para a análise qualitativa dos documentos referentes ao processo de aprimoramento do manual produzidos nos encontros grupais, foi adotado o método do Discurso do Sujeito Coletivo (DSC). Conforme o método do DSC, o estudo seguiu as seguintes etapas: leitura dos registros com a submissão destes a um trabalho analítico inicial de decomposição; identificação das expressões-chave; identificação das ideias centrais; construção dos discursos individuais; recomposição do pensamento coletivo para a construção do DSC a partir dos diferentes discursos individuais (LEFÉVRE; LEFÉVRE, 2003).

Essa estratégia discursiva permite explicitar uma dada representação social, bem como o conjunto 
das representaçóes que formam o imaginário social (LEFÉVRE; LEFÉVRE, 2003) - neste caso, referentes às experiências vivenciadas no contexto de saúde $\mathrm{e}$ social pelas pessoas com EM participantes do grupo.

\section{Resultados}

O grupo desenvolvido pela Terapia Ocupacional envolveu um total de 10 pessoas com diagnóstico de EM, das quais três homens e sete mulheres, com idade entre 23 e 55 anos. O tempo de doença variou de 1 a 19 anos, tendo a grande maioria cinco anos ou mais de tempo de doença. Todos apresentavam autonomia para as atividades diárias, com variaçáo do EDSS de nenhuma incapacidade a incapacidade moderada. A escolaridade variou de ensino médio completo a ensino superior completo. Quanto à ocupaçáo, cinco trabalhavam, um participante era do lar e quatro estavam aposentados. Todos realizavam tratamento no Ambulatório de Doenças Desmielinizantes do IC-HC da FMUSP.

Na versão preliminar do "Manual de Orientaçóes para Pessoas com Esclerose Múltipla”, constavam informaçóes gerais quanto à doença e orientaçóes sobre fadiga, memória, equilíbrio e organizaçáo das atividades rotineiras. A discussão do conteúdo do manual ocorreu no contexto de atenção grupal, coordenado pela Terapia Ocupacional, a partir de questôes disparadoras, tais como: necessidades percebidas no dia a dia, situaçóes de vida dos participantes, incapacidades percebidas e possibilidades de utilizaçáo do manual por parte dos participantes e de sua rede de suporte. Isso conduziu a uma leitura crítica do manual, à sua adequação quanto ao conteúdo e linguagem, e à avaliação quanto à sua aplicabilidade pelos componentes do grupo.

No processo de aprimoramento do manual em grupo, valorizou-se a perspectiva dos participantes, com sugestóes quanto à forma e ao conteúdo, tais como: 1- seleçáo de novas imagens diretamente relacionadas às orientaçóes; 2- organização das informaçóes por agrupamento temático; 3 - inclusão de novas informaçóes; 4- substituição e inclusão de termos que fossem mais claros e objetivos, e, assim, facilitassem a compreensão e o uso do manual.

A versão final apresentou informaçôes sobre a doença e orientaçóes para o dia a dia, relacionadas a diferentes conteúdos: gerenciamento da fadiga, conservação de energia, recursos para a memória, orientaçôes quanto ao equilíbrio corporal, organização das atividades de rotina, manutenção da temperatura corporal e outros cuidados. A linguagem simples e objetiva, assim como o uso de recursos pictográficos, facilitou a compreensão da informação e a consulta do manual por subdivisão temática. A Figura 2 ilustra

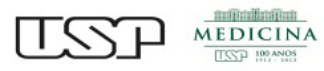

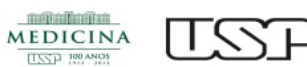

\section{Fadiga}

- Escolha uma cama com altura adequada para facilitar o momento de levantar e deitar;

- Retire os tapetes para evitar que escorregue ou tropece;

- Escolha calçados que tragam estabilidade ao seu caminhar.

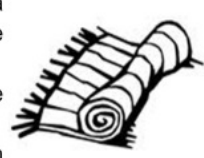

\section{Dificuldade de memória}

A memória é uma função importante para a organização do dia-a-dia. Dificuldades de memória podem dificultar a realização das tarefas de casa, trabalho e tomadas de decisões.

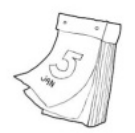

- Use agendas para marcar todos os seus compromissos e obrigações;

- Faça uma lista com todas as tarefas do seu dia-a-dia;

- Coloque etiquetas de identificação nos armários contendo os objetos guardados;

- Anote as atividades realizadas e ideias em um diário;

- Faça o uso de despertadores para lembrar compromissos, remédios e tarefas;

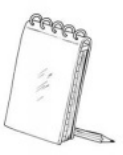

Acadêmicas de Terapia Ocupacional Paula Pozzi Pimentel e Marina Bolognani Terapeuta Ocupacional Ana Cristina F. Souto Prof Dr. Rosé Colom Toldrá

A fadiga é uma sensação de profundo cansaço físico, mental e perda de energia, que muitas pessoas sofrem de tempos em tempos.

$\mathrm{Na} \mathrm{EM}$, o tipo de fadiga é mais intenso que simplesmente sentir-se cansado. A EM pode afetar a força e a coordenação dos movimentos, exigindo mais energia e tempo do que o habitual para fazer as atividades de rotina.

Essas atividades diárias podem ser planejadas para que tarefas simples e rotineiras não se tornem obstáculos ou causem fadiga.

A seguir encontram-se algumas ideias úteis e orientações para lidar $\mathrm{cm}$ os efeitos da fadiga, planejar as atividades, poupar esforços e contribuir para a manutenção de uma vida ativa.

\section{Dicas de saúde}

- Uma boa noite de sono ajuda a aliviar a fadiga física e mental;

- Uma dieta equilibrada, com orientação profissional, auxiliam na manutenção do peso;

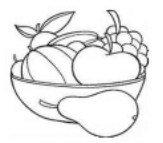

- As atividades físicas moderadas ajudam a manter a disposição, liberar tensões, relaxar e na manutenção do peso;

Figura 2. Orientações do "Manual de Orientações para Pessoas com Esclerose Múltipla" (PIMENTEL et al., 2013). 
algumas informaçôes e orientaçôes que constaram na versão final do "Manual de Orientaçóes para Pessoas com Esclerose Múltipla” (PIMENTEL et al., 2013).

O emprego do DSC para a análise qualitativa do processo de aprimoramento do manual gerou quatro ideias centrais e quatros respectivos Discursos do Sujeito Coletivo (DSCs), os quais assinalam a aplicabilidade do manual, conforme apresentado na Tabela 1. De acordo com o método, os discursos e as expressôes-chave são apresentados em itálico e na primeira pessoa no singular para indicar a pessoa falando (LEFÉVRE; LEFÉVRE, 2003).

\section{Discussão}

O estudo desenvolvido se insere na assistência de média complexidade, a qual envolve promoção da saúde, prevenção das doenças ou danos, tratamento e reabilitação, visando ao autocuidado (BRASIL, 2007). Dentre as estratégias, considera-se que o acesso à informação, comunicação e orientação são ações fundamentais para a autonomia e protagonismo das pessoas (BRASIL, 2004).

As diferenças entre os participantes do grupo quanto ao tempo de doença, idade, grau de incapacidade e experiência profissional demonstraram-se favoráveis para o compartilhamento de informaçôes e experiências. As percepçóes dos participantes sobre a versão preliminar do manual, a partir das questóes disparadoras, permitiu a livre expressáo dos participantes do grupo sobre a temática, o que levou à produção de um discurso representativo (LEFÉVRE; LEFÉVRE, 2003).

Desse modo, o aprimoramento do manual em grupo e de forma dialogada originou uma ferramenta de fácil consulta, em linguagem simples e objetiva, visando sensibilizar e estimular a adoção de práticas de autocuidado e de promoção da saúde (BRASIL, 2012, 2014). Também a condução do grupo de forma participativa, em busca de soluçóes coletivas, propiciou a compreensão das próprias questóes, superação de problemas, de forma que o manual se tornou um recurso complementar disponível para ajudar na tomada de decisóes relacionadas aos próprios cuidados (REBERTE; HOGA; GOMES, 2012; MOREIRA; NÓBREGA; SILVA, 2003).

A abordagem comunicativa e coletiva adotada é recomendada no processo de construçáo de uma cartilha educativa (REBERTE; HOGA; GOMES, 2012). A interação e a troca de conhecimento promovida durante o aprimoramento do manual, a partir do diálogo produzido, foram essenciais nesse processo devido a correspondência dos interesses e das necessidades apresentadas pelos participantes.

Segundo Zombini e Pelicioni (2011), a construçáo de um material educativo busca compilar conhecimento científico na literatura especializada, assuntos selecionados e informações necessárias para uma

Tabela 1. Ideias centrais e Discursos do Sujeito Coletivo (DSCs) dos participantes do grupo sobre o manual de orientações, São Paulo, 2016.

\begin{tabular}{|c|c|}
\hline Ideia central & DSC \\
\hline $\begin{array}{c}\text { O manual ajuda a } \\
\text { desmistificar a doença e } \\
\text { aprender a se cuidar. }\end{array}$ & $\begin{array}{l}\text { O manual explica as dúvidas para quem descobriu recentemente a doença e não } \\
\text { tem muita informação. Traz uma boa base para entender os sintomas e explica } \\
\text { para os familiares e amigos o que é a doença. Esclarece sobre o diagnóstico e tira } \\
\text { aquele medo... de ficar de cadeira de rodas elou em outras situações muito graves } \\
\text { de saúde. Explica a vivência de quem tem EM e situações diferentes que você não } \\
\text { sabe como resolver, como o cansaço. Com informaçâ as pessoas entendem sobre } \\
\text { o assunto e passam a se cuidar mais. }\end{array}$ \\
\hline O manual é um guia. & $\begin{array}{l}\text { É um guia... as dicas da rotina de casa, como a altura dos varais e uso do tapete, } \\
\text { sugestões práticas que são boas. O manual me ajudou a montar minha rotina... } \\
\text { como dividir as tarefas, colocar nomes nos locais para identificação, adiantar o } \\
\text { preparo dos alimentos para poupar tempo e energia, quando estiver com fadiga e } \\
\text { adequar à série de exercícios e movimentos. }\end{array}$ \\
\hline $\begin{array}{l}\text { O manual serve como } \\
\text { apoio. }\end{array}$ & $\begin{array}{l}\text { O manual ajuda quando me irrito com as situações e dificuldades provenientes da } \\
\text { doença. Utilizo o manual para uma identificaçáo com os problemas que aparecem e } \\
\text { tento estar aberta para compreender melhor. É como se alguém estivesse contando } \\
\text { que tem os mesmos problemas que eu tenho. Melhora a autoestima e fala das } \\
\text { preocupações com o corpo. }\end{array}$ \\
\hline $\begin{array}{l}\text { O manual facilita a } \\
\text { convivência. }\end{array}$ & $\begin{array}{l}\text { O manual até melhorou a relação com a minha mulher, pois abaixa a ansiedade. } \\
\text { Ela ficou mais compreensiva e mais confortável com as informações. Minha filha } \\
\text { achou bom, pois há dicas que faltavam nos outros materiais de EM. Meus familiares } \\
\text { disseram que o manual está ótimo, para parentes, amigos e pessoas com EM. }\end{array}$ \\
\hline
\end{tabular}


abordagem significativa, de forma compreensível e convidativa ao público destinado. Somado a isso, a estratégia de aprimoramento em situação grupal e a estratégia do uso do DSC valorizaram a opiniāo e as vivências decorrentes da doença, assim como o processo de negociação de significados e a valorizaçáo de experiências entre profissionais de saúde e usuários. Tais processos são considerados importantes para a melhoria da qualidade do material, para fins educativos (PANOBIANCO et al., 2009; ZOMBINI; PELICIONI, 2011).

No material educativo, deve-se procurar empregar mecanismos que reduzam as barreiras de compreensão da mensagem com linguagem simples e recursos pictográficos, minimizando as dificuldades de comunicação e tornando-o mais eficiente (MOREIRA; NÓBREGA; SILVA, 2003) e mais atrativo para o leitor, à medida que desperta o interesse e auxilia na compreensão do texto (TORRES et al., 2009). Em consonância, neste estudo, identificou-se que o material aprimorado se apresentou ilustrado, de fácil entendimento e com orientações que estimularam a realização das atividades cotidianas, a autonomia, o protagonismo e a promoçáo da qualidade de vida.

Conforme encontrado nos diferentes DSCs, o aprimoramento do manual, o seu uso e a discussão gerada em torno do "Manual de Orientaçóes para Pessoas com Esclerose Múltipla” permitiram a reflexão sobre os próprios sintomas, sobre as dificuldades enfrentadas, e estimularam a corresponsabilidade com o autocuidado, aspecto importante no caso de doenças crônicas. Isso reforça a ideia de que propostas centradas nas pessoas e em suas necessidades permitem conduzir as atividades grupais com a colaboração e o comprometimento dos participantes (MALTA; MERHY, 2010). Assim, a prática "educativo-dialógica" possibilitou mudanças no estilo de vida, na medida em que os participantes se identificaram como agentes de transformação e ampliaram a compreensão sobre os problemas a partir da reflexão (TORRES et al., 2009).

O curso crônico-progressivo e imprevisível da doença demanda flexibilidade para o enfrentamento dos sintomas, das possíveis incapacidades no cotidiano e a adoção de práticas de autocuidado. Dentre as estratégias, o gerenciamento da fadiga e as mudanças na forma de realizaçáo das atividades cotidianas constituíram-se recursos importantes para o autocuidado e a promoção de saúde (TOLDRÁ, 2013). Tais informaçóes no manual enfatizaram a importância do equilíbrio na realização das atividades do dia a dia, o que proporcionou às pessoas maior condição de lidar com os fatores que interferiam no seu autocuidado e na sua rotina (PANOBIANCO et al., 2009).

Evidenciaram-se nos DSCs a validade do manual no dia a dia e na sensibilização da rede social, como familiares, por facilitar a compreensão da doença e de suas repercussôes nas relaçôes interpessoais. Isso permite uma melhor comunicação sobre a doença, desmistificação de sintomas e entendimento sobre as dificuldades enfrentadas pelas pessoas, ajudando na ampliação do suporte no dia a dia (OLIVEIRA; FERNANDES; SAWADA, 2008).

A incorporação de materiais educativos nas atividades dos profissionais marca a importância prática dos assuntos abordados, o que facilita o processo de orientação em saúde. Também, o uso de um material educativo pela equipe de saúde unifica a linguagem, bem como proporciona a integralidade e a multidisciplinaridade do cuidado, conforme preconiza o Sistema Único de Saúde (SUS) (ZOMBINI; PELICIONI, 2011; BRASIL, 2014). Segundo Silva e Cardoso (2009), além de uma ferramenta para esclarecimento sobre aspectos técnicos referentes às doenças e terapêuticas, os manuais educativos também se apresentam como um eficiente instrumento de interação entre a equipe de saúde e usuários, possibilitando um modo objetivo de transmitir informaçôes com vistas à promoção da saúde.

Dessa forma, a versão preliminar do manual, construída a partir das informaçóes das próprias pessoas com EM, de terapeutas ocupacionais e dos médicos do IC-HC da FMUSP, fomentou uma ação educativa que possibilitou a permuta de experiências e informações, e, posteriormente, estimulou a reflexão de temas para o aprimoramento do manual no contexto do grupo. Esse processo permitiu validar as experiências de cada um dos atores participantes como fonte de conhecimento (TORRES et al., 2009).

Os manuais de orientação, em geral, buscam contemplar as experiências profissionais e responder aos questionamentos e às dúvidas mais frequentes dos usuários. Tais informaçôes devem auxiliar na tomada de decisóes e prevenir comportamentos e atitudes que possam ser prejudiciais à saúde (GOZZO et al., 2012). O formato impresso viabiliza que a pessoa leve o material para casa, o que permite maior adesão às orientações (SANTOS et al., 2012), uma vez que possibilita leituras posteriores e reflexóes sobre o conteúdo. Em consonância com os autores, os DSCs revelaram o papel facilitador do manual em relação ao enfrentamento das dificuldades decorrentes da $\mathrm{EM}$, aos esclarecimentos de dúvidas e à prática da aprendizagem. 
A grande contribuição do material educativo escrito no contexto da educação em saúde é atender às necessidades das pessoas (MOREIRA; NÓBREGA; SILVA, 2003). Dessa forma, o "Manual de Orientação para Pessoas com Esclerose Múltipla" (PIMENTEL et al., 2013), aprimorado no grupo, conforme os DSCs, passou a ser utilizado como uma ferramenta útil no dia a dia pelo fácil acesso ao aprendizado adquirido no grupo, o que contribuiu para o autocuidado e a promoção da saúde.

\section{Conclusão}

A elaboração de um manual de orientação para pessoas com EM, em consonância com as políticas do SUS, permitiu o desenvolvimento de uma importante ferramenta para a promoção da corresponsabilização pelo autocuidado, visando à promoção da saúde.

As etapas de elaboração e de aprimoramento do manual de orientação contribuíram para a construção de um material que somou e validou as experiências e conhecimentos de usuários, profissionais e familiares, obtendo como resultado um material pertinente e direcionado à realidade das pessoas com EM.

Segundo os DSCs, o manual mostrou-se uma ferramenta adequada, útil e prática para o oferecimento de informação e orientação a serem incorporados no dia a dia pelas pessoas com EM. Ademais, sua aplicação pode estender-se aos familiares e aos demais membros da rede de suporte social, favorecendo a ampliação de apoio a essas pessoas.

Como limite do estudo, aponta-se para o fato de este ter sido constituído com um único grupo. Ressalta-se a importância de realização de outras pesquisas que possam instrumentalizar o desenvolvimento de manuais educativos no âmbito do autocuidado, bem como fomentar o seu uso para potencializar as abordagens terapêuticas adotadas pelos profissionais das diferentes áreas.

\section{Referências}

BRASIL. Ministério da Saúde. Humaniza SUS. Politica Nacional de Humanização: a humanização como eixo norteador das práticas de atenção e gestão em todas as instâncias do SUS. Brasília, 2004. Disponível em: <http://bvsms.saude.gov.br/bvs/publicacoes/humanizasus_2004.pdf>. Acesso em: 1 jun. 2012.

BRASIL. Conselho Nacional de Secretários de Saúde - CONASS. Assistência de média e alta complexidade no SUS. Brasília, 2007. Disponível em: <http://bvsms. saude.gov.br/bvs/publicacoes/colec_progestores_livro9. pdf>. Acesso em: 1 jun. 2012.
BRASIL. Ministério da Saúde. Portaria no 793, de 24 de abril de 2012. Institui a Rede de Cuidados à Pessoa com Deficiência no âmbito do Sistema Único de Saúde. Diário Oficial [da] República Federativa do Brasil, Poder Executivo, Brasília, DF, 24 jul. 2012. Disponível em: <http://bvsms.saude.gov.br/bvs/saudelegis/gm/2012/ prt0793_24_04_2012.html>. Acesso em: 11 jul. 2014.

BRASIL. Ministério da Saúde. Secretaria de Vigilância à Saúde. Secretaria de Atenção à Saúde. Politica Nacional de Promoção da Saúde: PNaPS: revisão da Portaria MS/ GM no 687, de 30 de março de 2006. Brasília, 2014. Disponível em: <http://bvsms.saude.gov.br/bvs/publicacoes/politica_nacional_promocao_saude_pnaps.pdf>. Acesso em: 11 jul.2015.

CALLEGARO, D.; LINO, A. M. M.; MARCHIORI, P. E. Esclerose múltipla e outras doenças desmielinizantes do sistema nervoso central. In: MARTINS, M. A. et al. Clínica médica. Barueri: Manole, 2009. p. 449-478.

FRAGOSO, Y. D. et al. The effect of multiple sclerosis on the professional life of a group of Brazilian patients. Arquivos de Neuro-Psiquiatria, São Paulo, v. 68, n. 6, p. 914-917, 2010. http://dx.doi.org/10.1590/S0004$-282 \times 2010000600016$

GARCIA-BURGUILLO, M. P.; AGUILA-MATURANA, A. M. Energy-saving strategies in the treatment of fatigue in patients with multiple sclerosis. Revista de Neurologia, Barcelona, v. 49, n. 4, p. 181-185, 2009.

GIL, A. C. Métodos e técnicas de pesquisa social. São Paulo: Atlas, 2006.

GOZZO, T. O. et al. Informaçóes para mulheres com câncer de mama. Escola Anna Nery Revista de Enfermagem, Rio de Janeiro, v. 16, n. 2, p. 306-311, 2012.

HASSELKUS, B. R. Introdução às populaçôes de adultos e idosos. In: NEISTAD, T. M. E.; CREPEAU, E. B. Willard \& Spackman: Terapia Ocupacional. Rio de Janeiro: Guanabara Koogan, 2002. p. 607-615.

LEFÉVRE, F.; LEFÉVRE, A. M. C. O Discurso do Sujeito Coletivo: um novo enfoque em pesquisa qualitativa. Caxias do Sul: EDUCS, 2003. (Desdobramentos).

LÜDKE, M.; ANDRÉ, M. Pesquisa em educação: abordagens qualitativas. São Paulo: Pedagógica e Universitária, 1988.

MALTA, D. C.; MERHY, E. E. O percurso da linha do cuidado sob a perspectiva das doenças crônicas não transmissíveis. Interface - Comunicação, Saúde, Educação, Botucatu, v. 14, n. 34, p. 593-605, 2010. http://dx.doi. org/10.1590/S1414-32832010005000010.

MENDES, M. F. et al. Fadiga na forma remitente recorrente da esclerose múltipla. Arquivos de Neuro-Psiquiatria, São Paulo, v. 58, n. 2B, p. 471-475, 2000.

MOREIRA, M. F; NÓBREGA, M. M. L.; SILVA, M. I. T. Comunicação Escrita: contribuição para a elaboração de material educativo em saúde. Revista Brasileira de En- 
fermagem, Brasília, v. 56, n. 2, p. 184-188, 2003. http:// dx.doi.org/10.1590/S0034-71672003000200015.

NOGUEIRA, L. A. C. et al. The effect of functional limitations and fatigue on the quality of life in people with multiple sclerosis. Arquivos de Neuro-Psiquiatria, São Paulo, v. 67, n. 3B, p. 812-817, 2009.

OLIVEIRA, M. S.; FERNANDES, A. F. C.; SAWADA, N. O. Manual educativo para o autocuidado da mulher mastectomizada. Texto \& Contexto - Enfermagem, Florianópolis, v. 17, n. 1, p. 115-23, 2008.

PANOBIANCO, M. S. et al. Construção do conhecimento necessário ao desenvolvimento de um manual didático-instrucional na prevenção do linfedema pós-mastectomia. Texto \& Contexto - Enfermagem, Florianópolis, v. 18, n. 3, p. 418-26, 2009.

PIMENTEL, P. P. et al. Manual de orientaçóes para pessoas com esclerose múltipla. São Paulo: FMUSP, 2013.

REBERTE, L. M.; HOGA, L. A. K.; GOMES, A. L. Z. $\mathrm{O}$ processo de construçáo de material educativo para a promoção da saúde das gestantes. Revista Latino-Americana de Enfermagem, São Paulo, v. 20, n. 1, p. 101-108, 2012.

SANTOS, A. M. B.; TATIANA, P. O.; PIEMONTE, M. E. P. Elaboração de um manual ilustrado de exercícios domiciliares para pacientes com hemiparesia secun- dária ao acidente vascular encefálico (AVE). Fisioterapia e Pesquisa, São Paulo, v. 19, n. 1, p. 2-7, 2012.

SILVA, G. R. F.; CARDOSO, M. V. L. M. L. Percepção de mães sobre um manual educativo sobre estimulação visual da criança. Revista Eletrônica de Enfermagem, Goiânia, v. 11, n. 4, p. 847-57, 2009.

TOLDRÁ, R. C. Terapia Ocupacional e o Método Self-Healing: criando possibilidades de viver o corpo. In: PÁDUA, E. M. M.; MAGALHÃES, L. V. Casos, memórias e vivências em Terapia Ocupacional. Campinas: Papirus, 2005. p. 97-114.

TOLDRÁ, R. C. Autocuidado e prevenção de incapacidades para pessoas com esclerose múltipla e outros diagnósticos: aperfeiçoando e ampliando sua utilização. São Paulo: Rettec Artes Gráficas e Editoria, 2013. Catálogo Projetos 2012-2013 Aprender com Cultura e Extensão.

TORRES, H. C. et al. O processo de elaboração de cartilhas para orientação do autocuidado no programa educativo em diabetes. Revista Brasileira de Enfermagem, Brasília, v. 62, n. 2, p. 312-316, 2009. http://dx.doi. org/10.1590/S0034-71672009000200023.

ZOMBINI, E. V.; PELICIONI, M. C. F. Estratégias para a avaliação de um material educativo em saúde ocular. Revista Brasileira de Crescimento e Desenvolvimento Humano, São Paulo, v. 21, n.1, p. 51-58, 2011.

\section{Contribuição dos Autores}

Paula Pozzi Pimentel: Responsável pela elaboração de registros/transcrição/ análise dos documentos e redação do texto. Rosé Colom Toldrá: Responsável pela concepção/condução da pesquisa, participação na análise e revisão final do texto. Ambas as autoras aprovaram a versão final do texto.

\section{Notas}

${ }^{1}$ Este material é parte da pesquisa "Atenção à saúde e reabilitação de média complexidade à pessoa com deficiência física no âmbito da terapia ocupacional: perfil, demandas e açóes". 